\title{
ESTUDIOS FENOMORFOLÓGICOS EN LA VEGETACIÓN DEL SUR DE ESPAÑA. III. COMPORTAMIENTO ESTACIONAL DE UNA COMUNIDAD DE BADLANDS (TABERNAS, ALMERÍA. ESPAÑA)
}

\author{
Teresa NAVARRO, José M. NIETO CALDERA, \\ Andrés V. PÉREZ LATORRE y Baltasar CABEZUDO
}

\begin{abstract}
RESUMEN. Estudios fenomorfológicos en la vegetación del sur de España. III. Comportamiento estacional de una comunidad de badlands (Tabernas, Almería, España). Se describe el comportamiento fenomorfológico de una comunidad de badlands del sureste de la Península Ibérica. Se ha realizado un análisis basado en el estudio de las distintas fenofases para los táxones persistentes de la comunidad. Para cada especie se tomaron los datos de inicio y duración de cada fenofase (prefloración, floración, fructificación, dispersión, caida de hojas, crecimiento vegetativo). Se caracterizan las estaciores más favorables o desfavorables para el ciclo biológico de las especies de la comunidad y la relación de las fenofases con el clima local. Se concluye que la comunidad estudiada presenta un comportamiento fenofásico multiestacional, siendo la primavera y el verano donde tienen lugar la mayor parte de los cambios fenofásicos.
\end{abstract}

Palabras clave. Fenomorfología, badlands, Almería, SE España.

\begin{abstract}
Phenomorphological studies in South of Spain vegetation. III. Seasonality of a badlands community. (Tabernas. Almería. Spain). Phenomorphological behauviour of a badlands community in the southwest ot Iberian Peninsula, is described in this work. An analysis based on the study of phenological phases for persisting species was carried out. Data for starting and maintenance of phenophases were taken for each species ( flower buds formation (PF), flowering (FL), fruit setting (FR), seed dispersal (DS), brachyblast vegetative growth (CVB), dolicoblast vegetative growth (CVD), leaf shedding brachyblast $(\mathrm{CHB})$ and leaf shedding dolicoblast (CHD)) More favourable or unfavourable seasons for biological cycle of the community species and the relation among phenological phases and local climate were characterized. As outcome, the studied community shows a multiseasonal phenological pattern. Spring and summer are the seasons where the greater part ot the phenological changes occur.
\end{abstract}

Key words. Pheno-morphology, badlands, Almería, SE Spain.

\section{INTRODUCCIÓN}

Es de gran interés la realización de estudios fenomorfológicos en la Región Medite- rránea (Orshan, 1964, 1989; Montenegro et al., 1979; Aljaro et al., 1981; Cabezudo et al., 1992) que permitan detectar los cambios estacionales de una planta en respuesta a su 
adaptación al ambiente (Orshan, 1982) y conocer, mediante el seguimiento de las fenofases de cada órgano, la dinámica morfofuncional de su ciclo anual. En el presente trabajo se analiza el comportamiento fenomorfológico de una comunidad vegetal de badlands en el sureste árido español y se detectan diferentes patrones de comportamiento fenológico de la especies en función de su localización topográfica.

El área de estudio se sitúa en el término municipal de Tabernas (Desierto de Tabernas, Almería. $2^{\circ} 08^{\prime} \mathrm{W}$ y $37^{\circ} 00^{\prime} \mathrm{N}$ ) a una altitud de $400 \mathrm{~m}$. La estación meteorológica más cercana corresponde a esta misma localidad, con observaciones termométricas de 16 años y con un registro de precipitaciones de 25 años. En la figura 1 se representa la distribución de dichos parámetros a lo largo del año, observándose que los meses húmedos son de noviembre a marzo. Allué (1990) define el clima como subdesértico subárido y Capel Molina (1990), según la clasificación de Koppen-Geiger, 1o define como seco estepario. Peinado et al., (1992) definen bioclimáticamente el termotipo como termomediterráneo superior de ombroclima semiárido inferior.

El badland es una formación geomorfológica característica de regiones de marcado contraste estacional, en las que existe un largo período seco. Geomorfológicamente, se distinguen tres zonas topográficas: cresta de la pendiente; cara de la pendiente, con

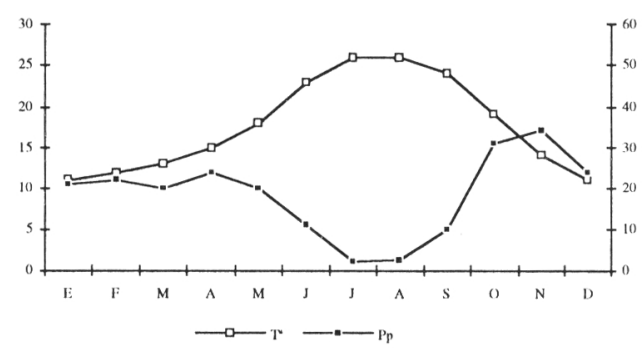

Figura 1. Diagrama ombrotérmico. Tabernas (Almería). Hydrothermic diagram. Tabernas (Almería). estrechos interfluvios de fino drenaje y pie de la cara de la pendiente, donde se deposita el escaso material de arrastre originado por la erosión laminar.

La comunidad estudiada queda reflejada en la tabla 1. Las especies ubicadas en la cresta de la pendiente, presentan fitosociológicamente afinidad por los matorrales de Anthyllido-Salsolion papillosae y Stipion tenacissimae. En la cara de la pendiente se desarrolla un grupo de especies incluibles en Salsolo-Peganion harmalae, de indudables apetencias nitrófilas y primocolonizadoras. Por último, en el pie de la cara de la pendiente, donde tienen lugar algunos fenómenos de hidromorfía y acumulación de sales, la vegetación está caracterizada por elementos de LygeoLimonion, Arthrocnemion y Suaedenion verae. Las especies estudiadas presentan diferentes adaptaciones morfofisiológicas (Blanca, 1991) como respuesta adaptativa al estrés climático (largos periodos secos) y geomorfológico (erosión laminar y escorrentía).

Tabla 1. Fenofases, afinidad fitosociológica, tipo biológico (Biot.) y situación topográfica (Top.) de las especies de la comunidad estudiada. Fenofases reproductivas: formación de yemas florales (PF), floración (FL), fructificación (FR) y dispersión (DS). Fenofases vegetativas: crecimiento vegetativo del dolicoblasto (CVD), crecimiento vegetativo del braquiblasto (CVB), caída de hojas del dolicoblasto (CHD), caída de hojas del braquiblasto (CHB). Tipo biológico: caméfito (Ca), hemicriptófito bianual $(\mathrm{H})$, nanocaméfito $(\mathrm{nCa})$, Caméfito/hemicriptófito $(\mathrm{Ca} / \mathrm{H})$, caméfito/ nanofanerófito $(\mathrm{Ca} / \mathrm{nF})$ y nanofanerófito $(\mathrm{nF})$. Phenological phases, phyto-sociological affinity, biological type (Biot.) and topographic position (Top.) for the species of the studied community. Reproductive phenophases: flower buds formation $(P F)$, flowering $(F L)$, fruit setting $(F R)$, seed dispersal (DS). Vegetative phenophases: brachyblast vegetative growth $(C V B)$, dolicoblast vegetative growth (CVD), leaf shedding brachyblast (CHB) and leaf shedding dolicoblast (CHD). Life form: chamaephyte $(\mathrm{Ca})$, hemicryptophyte $(H)$, nano-phanerophyte $(n F)$, nano-chamaephyte $(n C a)$. 


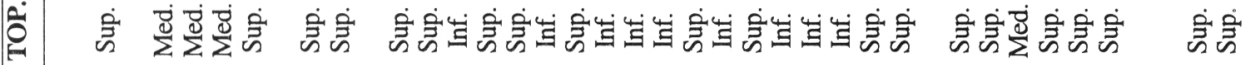

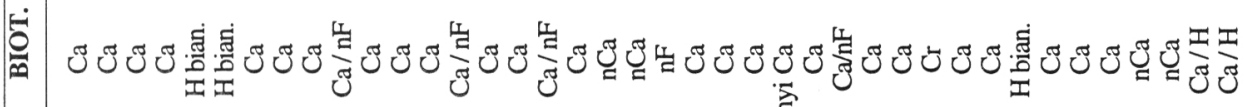

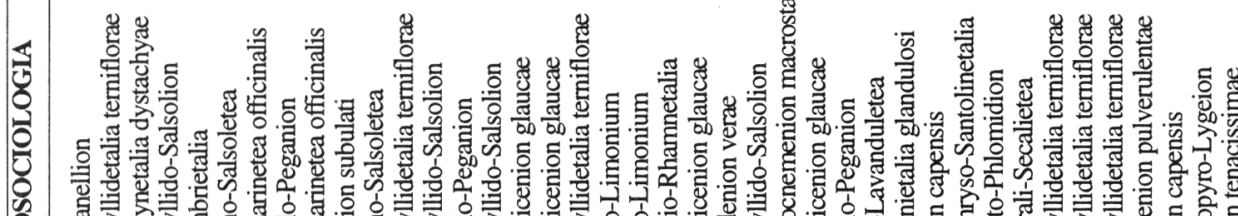

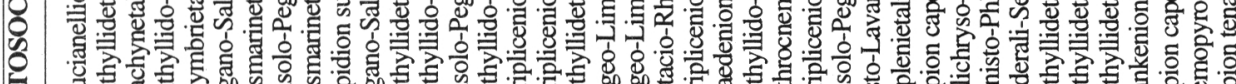

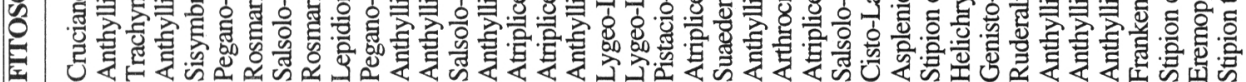

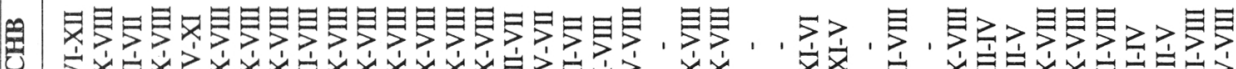

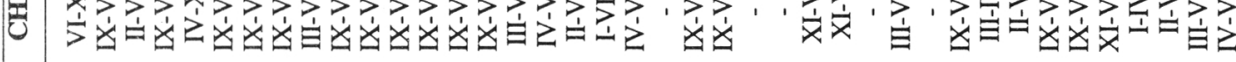

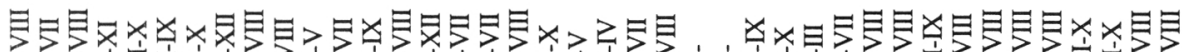

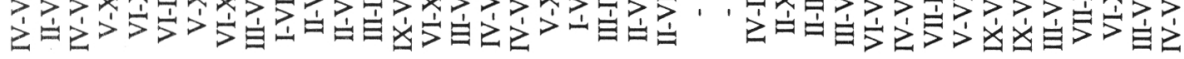

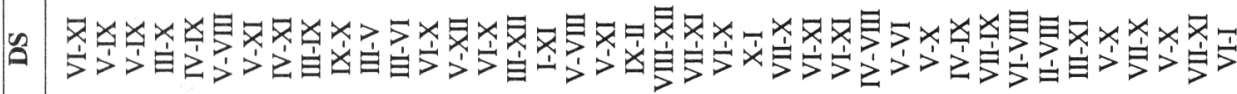

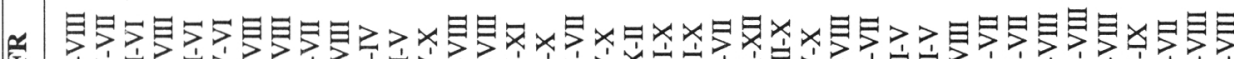

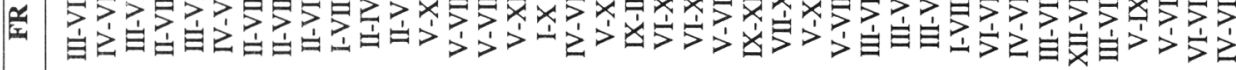
盂

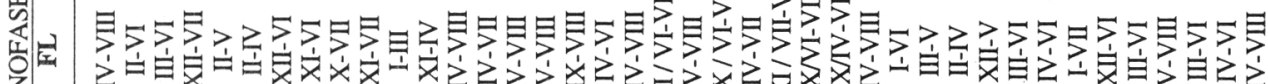
副

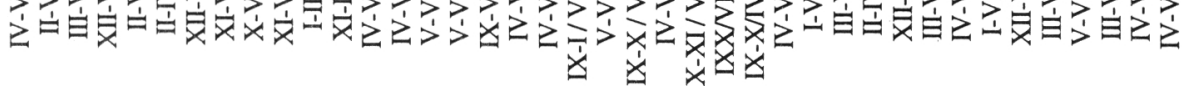

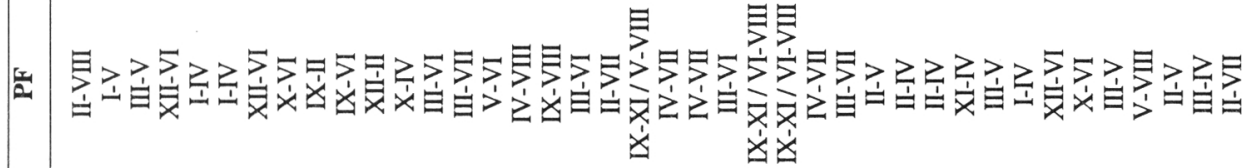

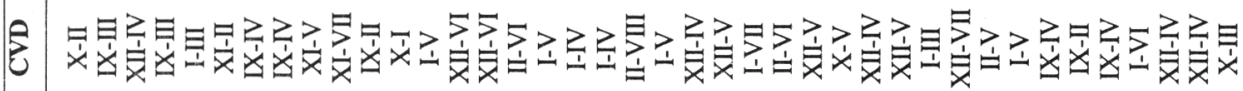

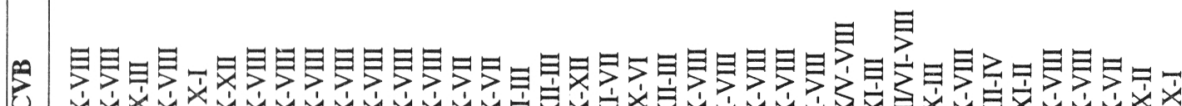

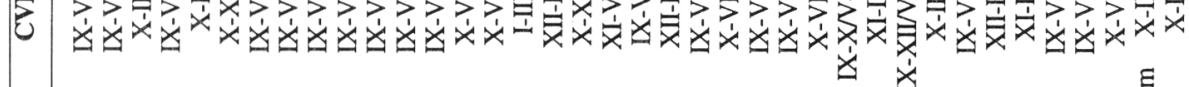

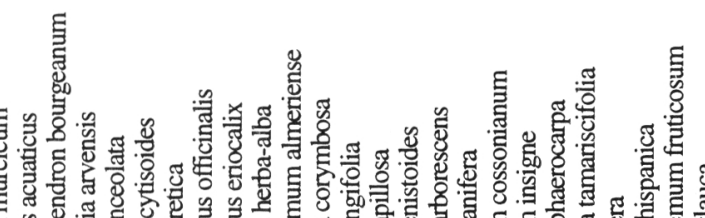

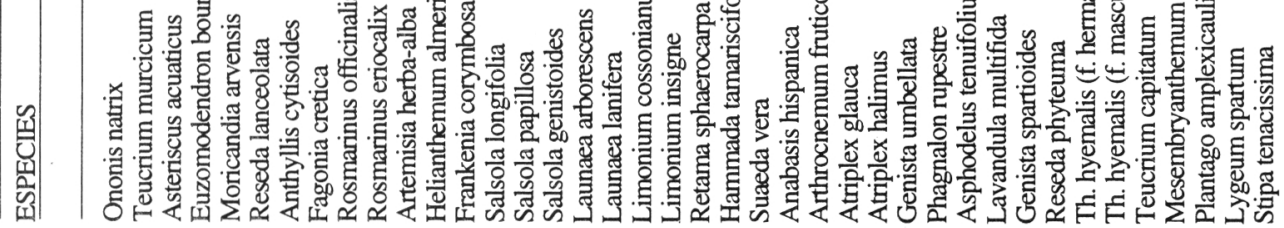




\section{MATERIAL Y MÉTODOS}

Se han estudiado un total de 39 táxones de diferentes comunidades de badlands. Son especies persistentes y siempreverdes; el tipo biológico predominante es el camefítico, típico de estepas y desiertos, (Raunkiaer, 1934 ; Cain, 1950), seguido de los caméfito/ nanofanerófitos y caméfito/hemicriptófitos.

Para la toma de datos se ha seguido el método de Orshan (1989). Cada especie, localizada en las diferentes zonas geomorfológicas del badland (tab. 1), ha sido observada durante 18 meses (Enero 1989 / Marzo 1990) con una periodicidad de dos semanas, obteniéndose un conocimiento cualitativo de las fenofases para cada estacionalidad. Se recolectaron partes representativas de la planta para la elaboración de un fenoherbario.

\section{RESULTADOS}

Se han obtenido resultados procedentes del análisis del comportamiento de la comunidad en función de los máximos observados para cada fenofase (Figs. 2 y 4 ) ; de las observaciones mensuales sobre el inicio y fin de cada una (Figs. 3 y 5, tab. 2); y de las diferencias fenofásicas respecto a la floración y crecimiento vegetativo de las especies según su localización topográfica en el badland. (figs. 6 y 7).

El óptimo de prefloración con más del $70 \%$ de las especies, corresponde a la primavera (marzo-abril). El 50\% posee una prefloración multiestacional (finales de invierno a principios de verano) y solo el $10 \%$ está en prefloración durante todo el año. La estación con mínimos de prefloración es otoño (septiembre) (fig. 2). El mes que registra el máximo de especies que inician la prefloración es marzo, seguido de febrero y enero. En verano (julio y agosto), no se registra ningún inicio de formación de yemas florales y en noviembre solo las forma Lavandula multifida (fig. 3).
Las especies con prefloración más prolongada son: Launaea arborescens, Limonium insigne, Frankenia corimbosa, Rosmarinus eriocaliyx y Thymus hiemalis.

El máximo de floración, más del $80 \%$ de las especies, es en primavera y verano. El 50\% están en flor unicamente en primavera (marzojunio) y el 10\% está en flor durante todo el año. Las estaciones con mínimos de floración son de finales de otoño a principios de invierno (fig. 2). En abril, inician la floración la mayoría de las especies, desde finales del verano a principios del otoño (agosto, septiembre y octubre) solo tres especies lo hacen: Atriplex halimus, Launaea arborescens y Rosmarinus officinalis (fig. 3). Launaea arborescens mantiene la floración durante la práctica totalidad del año. Artemisia herbaalba y Helianthemum almeriense poseen floración invernal y las especies del género Salsola unicamente estival. Hammada tamariscifolia, Anabasis hispánica, Artrhocnemum fruticosum y Atriplex glauca son multiestacionales de primavera, verano y otoño. Anthyllis cytisoides, Fagonia cretica, Rosmarinus officinalis, Euzomodendrum bourgeanum, Lavandula multifida y Thymus hyemalis son biestacionales de invierno y primavera.

El óptimo de fructificación, más de un $80 \%$ de especies, se registra de finales de primavera a principios de verano (mayo-junio). El $50 \%$ de la población está en fruto primavera y verano (abril a agosto). Solo el $5 \%$ fructifica todo el año (fig. 2). El mes donde se inicia la fructificación de la mayoría de las especies es mayo, seguido de marzo. En agosto, octubre y noviembre no lo hace ninguna especie (fig. 3). La fructificación más prolongada corresponde a Launaea arborescens, Rosmarinus eriocalix y Thymus hyemalis (funcionalmente hermafrodita).

La dispersión del $70 \%$ de especies es de verano a principios del otoño. El $50 \%$ de la población posee dispersión multiestacional (de 

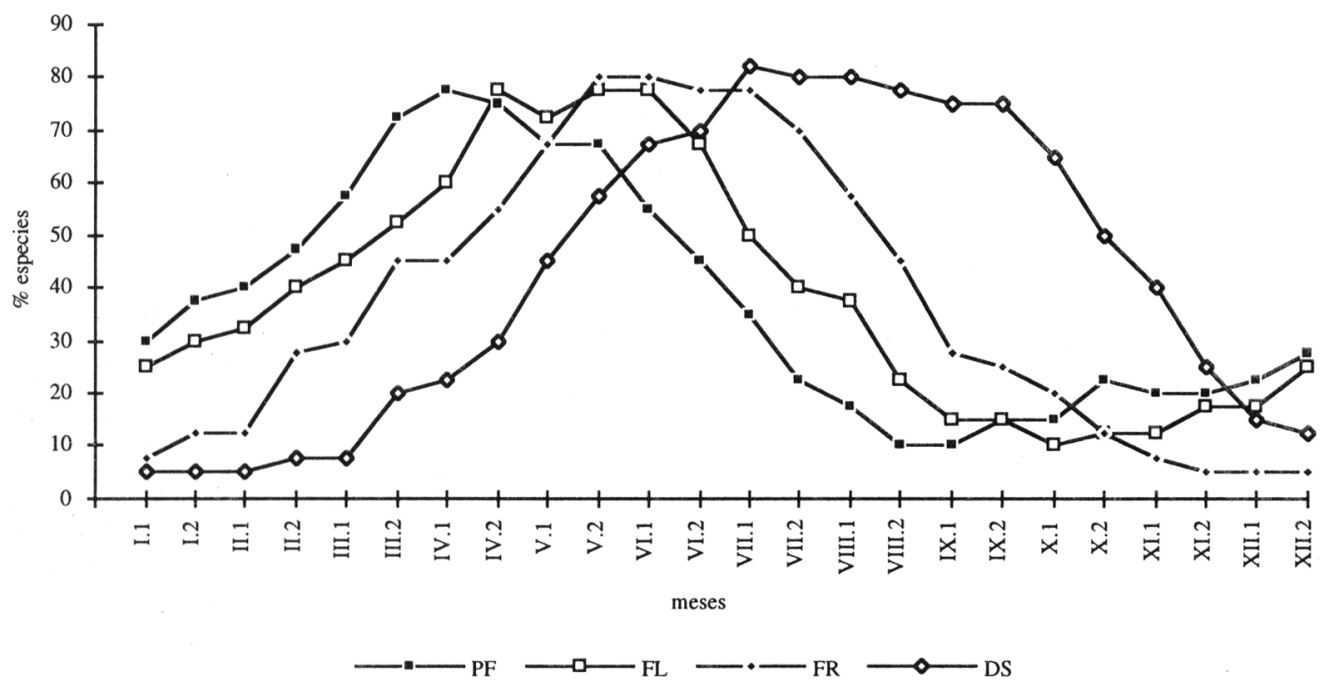

Figura 2. Porcentaje de especies que presentan las fenofases reproductivas en cada observación. Prefloración (PF), floración (FL), fructificación (FR) y dispersión (DS). Percentage of species showing reproductive phenophases each observation. Flower buds formation $(P F)$, flowering $(F L)$, fruit setting $(F R)$ and seed dispersal (DS).

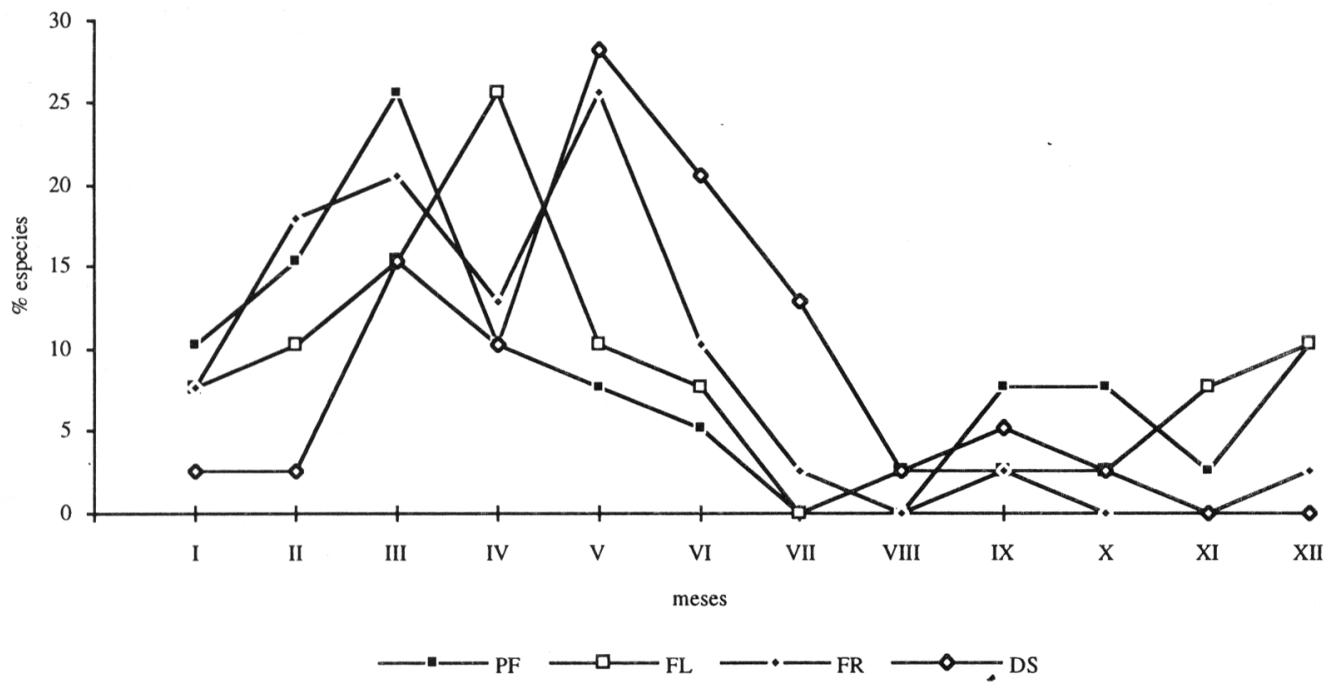

Figura 3. Porcentaje mensual de especies respecto al inicio de fenofases reproductivas. Prefloración (PF), floración (FL), fructificación (FR) y dispersión (DS). Monthly percentage of species starting reproductive phenophases. Flower buds formation $(P F)$, flowering $(F L)$, fruit setting $(F R)$ and seed dispersal $(D S)$. 


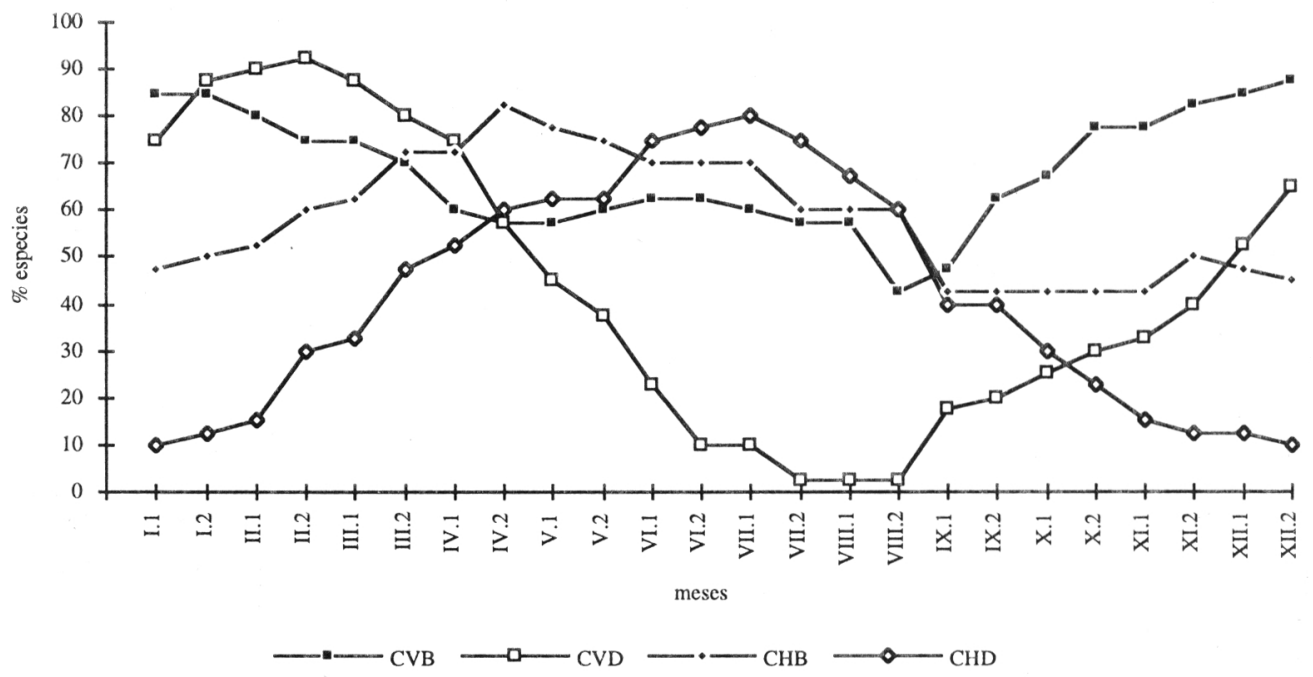

Figura 4. Porcentaje de especies que presentan las fenofases vegetativas cada observación. Crecimiento vegetativo del dolicoblasto (CVD), crecimiento vegetativo del braquiblasto (CVB), caída de hojas del dolicoblasto (CHD), caída de hojas del braquiblasto (CHB). Percentaje of species showing vegetative phenophases each observation. Dolichoblast vegetative growth (CVD), brachyblast vegetative growth $(C V B)$, leaf shedding dolichoblast $(C H D)$ and leaf shedding brachyblast $(C H B)$.

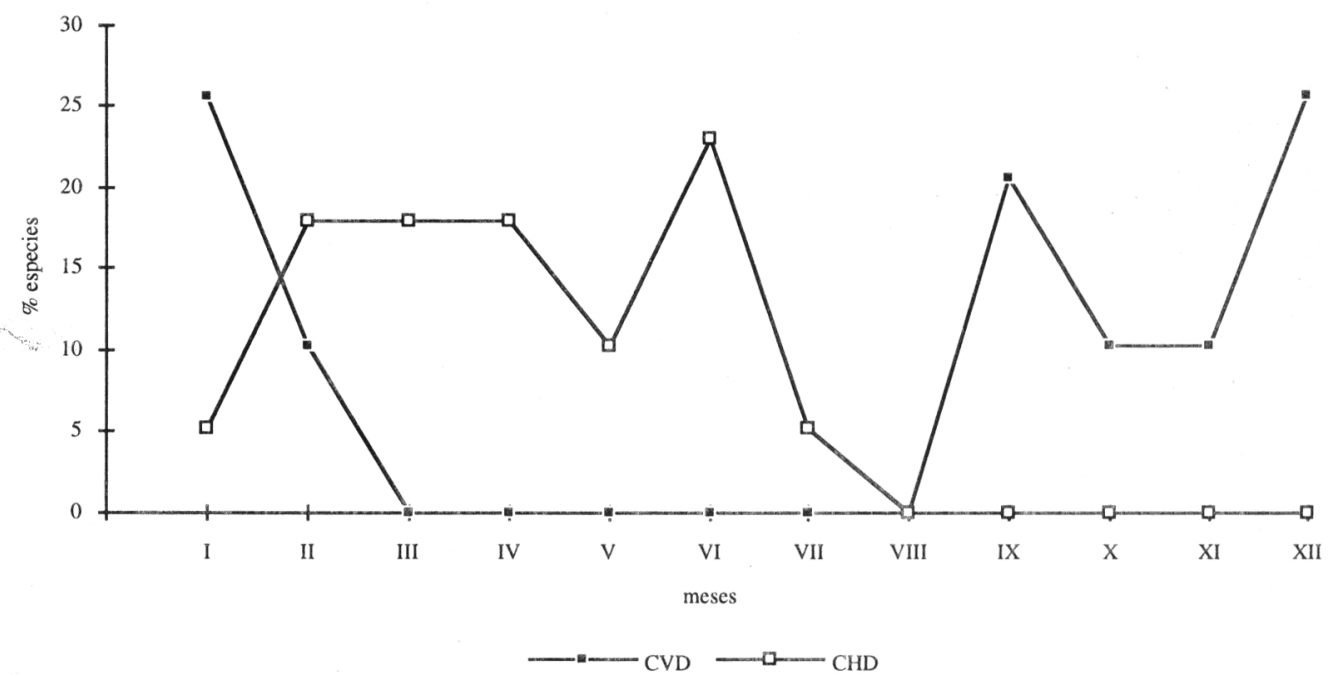

Figura 5. Porcentaje de especies respecto al inicio de fenofases vegetativas. Crecimiento vegetativo del dolicoblasto (CVD), caída de hojas del dolicoblasto (CHD). Percentaje of species starting vegetative phenophases. Dolichoblast vegetative growth (CVD), leaf shedding dolichoblast (CHD). 


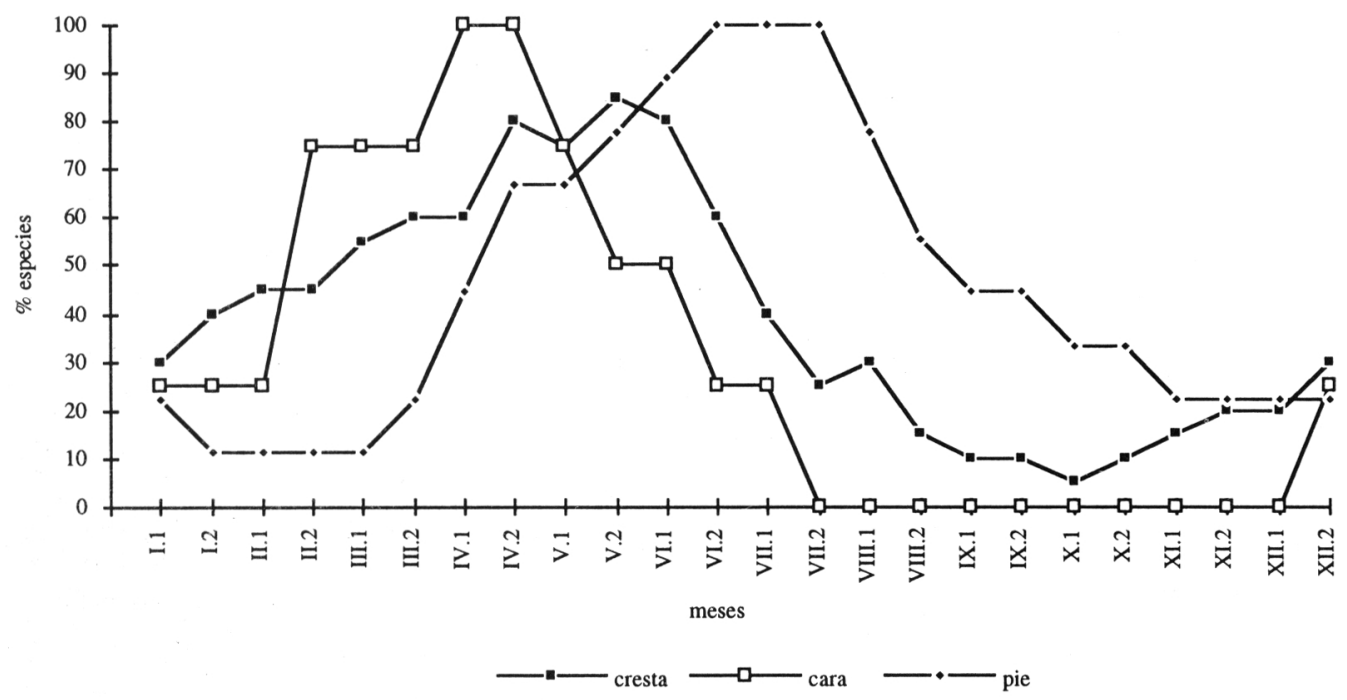

Figura 6. Porcentaje de especies que presentan floración en las tres zonas topográficas del badland (cresta, cara y pie de la pendiente) según observaciones mensuales. Percentage of species showing flowering in the three topographic zones of the badland (peak, slope and foot) according to fornigthly observations.

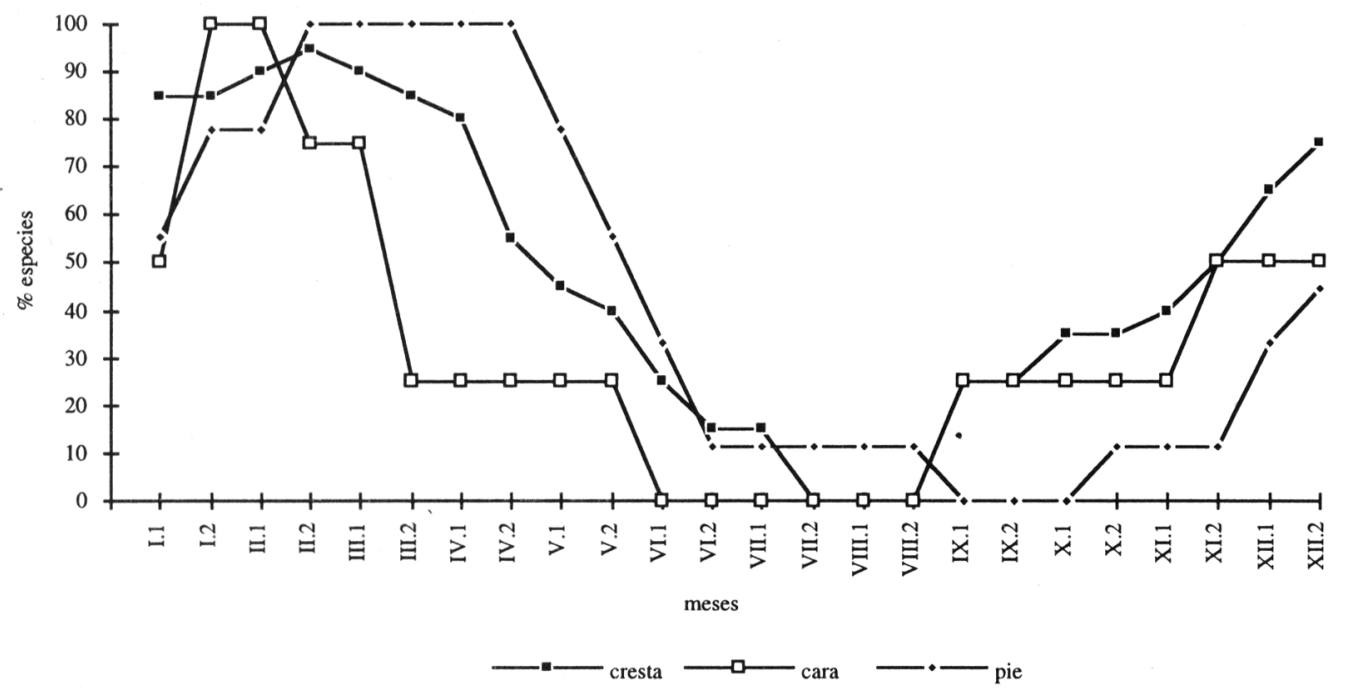

Figura 7. Porcentaje de especies que presentan crecimiento vegetativo en las tres zonas topográficas del badland (cresta, cara y pie de la pendiente) según observaciones mensuales. Percentage of species showing vegetative growth in the three zones of the badland (peak, slope and foot), according to fornigthly measurements. 


\begin{tabular}{ccccc}
\hline Fen. Rep. & PF & FL & FR & DS \\
\hline IF máx. & Pr. (III) & Pr. (IV) & Pr. (V) & Pr. (V) \\
IF mín. & Ve. (VII - VIII) & Ve. (VII) & Ve. - Ot. & In. (XII) \\
VF máx. & Pr. (III - IV) & Pr. - Ve. & Pr.- Ve. (IV - VII) & Ve. - Pr. - Ot. \\
VF mín. & Ot. (IX) & Ot. -In. & Ve. - Ot. & In. (I - II) \\
& & & & \\
\hline Fen. Veg. & CVD & CHD & CVB & CHB \\
\hline IF máx. & In. (XII_ I) & Ve. (VI) & Ot. (IX) & Pr. \\
IF mín. & Pr. - Ve. (III - VI) & Ot. & Ve. (VII - VIII) & Ot. \\
VF máx. & Ot. - Pr. & Ve. & In. - Pr. - Ot. & Pr. (IV) \\
VF mín. & In. (I - II) & Ot. & Ve. (VIII) & Ot. \\
\hline
\end{tabular}

Tabla 2. Caracterización anual (estaciones y meses) de las fenofases reproductivas (Fen. Rep.) y vegetativas (Fen. Veg.). IF: períodos con máximos (máx.) o mínimos (mín.) de iniciación de cada fenofase. VF: períodos con máxima (máx.) o mínima (mín.) concentración de especies en cada fenofase. Estaciones: invierno (In.), primavera (Pr.), verano (Ve.) y otoño (Ot.). Community anual characterization (seasons and months) based on reproductive (Fen. Rep.) and vegetative (Fen. Veg.) phenophases. IF: maximum (Max.) or minimum (min.) starting phenophase period. VF: maximum (max.) or minimum (min.) number of species showing each phenophase. In: Winter; Pr.: Spring; Ve: Summer; Ot: Autum.

primavera a otoño). Solo el 5\% está en dispersión todo el año. En invierno (enero y febrero), se registran los minimos de dispersión (fig. 2) y los máximos en mayo y junio. En diciembre no se dispersa ninguna especie (fig. 3). La dispersión más prolongada corresponde a Euzomodendron bourgeanum, Anthyllis cytisoides, Fagonia cretica, Stipa tenacissima, Limonium cossonianum, Thymus hyemalis (funcionalmente femenino) y las especies del género Salsola.

El óptimo de crecimiento vegetativo del dolicoblasto para la mayoría de las especies de la comunidad, se registra en invierno e inicio de la primavera. El 5\% de la población crece durante la práctica totalidad del año . El mínimo es en verano (fig. 4) y el máximo en invierno, seguido del inicio del otoño. No se detecta crecimiento de final de primavera y verano (fig. 5). Las especies con crecimiento vegetativo del dolicoblasto en la mayor parte de las estaciones del año son Anthyllis cytisoides, Frankenia corymbosa, Rosmarinus eriocalix, Lavandula multifida y Thymus hyemalis (funcionalmente hermafrodita). Las especies con crecimiento monoestacional son: Moricandia arvensis, Reseda lanceolata y Asphodelus tenuifolius.

El crecimiento vegetativo del braquiblasto es multiestacional, de otoño a inicios de primavera. El 50\% lo presenta durante todo el año. El mínimo corresponde al final del verano (fig. 4) y el máximo al inicio del otoño.

El período de máxima caída de hoja del dolicoblasto es en verano. El 50\% de las especies la arrojan en primavera y verano. El 10\% lo hace durante todo el año (fig. 4). La mayoría de las especies inician la caída en el verano pero otras lo hacen al final del invierno o inicios de la primavera. En otoño, esta fenofase no se inicia para ninguna especie (fig. 5).

Se han observado diferencias fenomorfológicas significativas del estudio comparativo del crecimiento vegetativo (fig. 6) y de la floración (fig. 7) de las especies según su localización topográfica en el badland. El crecimiento vegetativo del dolicoblasto en la cresta de la pendiente es de inicios de otoño a inicios de verano, en la cara de la pendiente es en invierno y en el pie de la pendiente de 
mediados de otoño a mediados de primavera. La floración en la cresta de la pendiente es biestacional, primavera y verano; en la cara de la pendiente, primaveral y en el pie de la cara de la pendiente estival.

\section{CONCLUSIONES}

El comportamiento fenofásico estacional de la comunidad estudiada (tab. 2) es de prefloración primaveral, floración y fructificación primaveral y estival, dispersión estival, crecimiento vegetativo del dolicoblasto invernal y primaveral, crecimiento vegetativo del braquiblasto otoñal, primaveral e invernal, caída de hoja del dolicoblasto estival y del braquiblasto primaveral. La prefloración, caída de hoja del dolicoblasto y braquiblasto son monoestacionales; la floración, fructificación, dispersión y crecimiento vegetativo del dolicoblasto son biestacionales y el crecimiento vegetativo del braquiblasto es multiestacional.

El inicio de las fases reproductivas de fructificación y de dispersión tiene lugar en primavera y el inicio del crecimiento vegetativo multiestacional del braquiblasto ocurre en otoño.

El crecimiento vegetativo de las ramas dolicoblasticas se efectúa en invierno y en primavera correlacionado con los máximos de precipitación. En primavera coincide con la prefloración, debido a la elongación de las ramas dolicoblasticas previa a la formación de las yemas florales. El crecimiento vegetativo del dolicoblasto guarda alta correlación con la precipitación, factor que afecta al crecimiento, siendo variable en función de los cambios climáticos anuales. En verano hay reducción del crecimiento vegetativo del dolicoblasto, por la sequia estival y las altas temperaturas que actuan como factores limitantes. El crecimiento vegetativo primaveral y estival de las ramas braquiblásticas, corresponde al dimorfismo estacional de las especies camefíticas, en las cuales hay caida y crecimiento de diferentes tipos de hojas y ramas en distintas estaciones del año (Orshan,1962 y 1964).

La floración coincide con el incremento de la temperatura. La caída de hoja se realiza en verano paralelamente a la sequía y las temperaturas elevadas que inducen en las plantas a una reducción del aparato vegetativo, por senescencia policárpica secuenciada con abcisión de hojas para los caméfitos-nano fanerófitos y con la caída de diferentes tipos de ramas, fundamentalmente dolicoblasticas, para los caméfitos y caméfito- hemicriptófitos.

En primavera, con el inicio de los aumentos de temperatura y precipitación, es cuando la mayor parte de las especies estudiadas presentan el crecimiento vegetativo y generativo.

En Thymus hyemalis la ginodioecia reproductiva establece diferencias en el comportamiento fenomorfológico, dado que los individuos funcionalmente hermafroditas poseen floración y fructificación más prolongada que los funcionalmente femeninos y estos mayor duración en el crecimiento vegetativo del dolicoblasto.

Los diferentes patrones de crecimiento vegetativo de las especies según su localización topográfica en el badland, sirven para comparar los grados de reducción vegetativa estacional de las especies según su tipo biológico y demostrar la plasticidad en el crecimiento vegetativo en areas desérticas, estrechamente vinculado al estrés geomorfológico. Los caméfitos de la cresta de la pendiente (ej. Helianthemum almeriense), presentan solo reducción estival del crecimiento vegetativo, ofreciendo una buena adaptación a los largos periodos secos por su dimorfismo estacional. Los caméfito-hemicriptófitos de la cara de la pendiente (ej. Moricandia arvensis ), con reducción del crecimiento durante todo el año a excepción del invierno, son menos resistentes a la sequedad prolongada que los caméfito- 
nanofanerófitos del pie de la pendiente (ej. Suaeda vera). Estos muestran una reducción del crecimiento vegetativo de mediados de primavera a mediados de otoño, con resistencia al periodo seco asociada a adaptaciones fisiológicas.

\section{BIBLIOGRAFÍA}

ALJARO, M.E., \& G. MONTENEGRO, -1981. Growth of dominant Chilean shrubs in the Andean Cordillera. Mountain Research and Developpment, 1(3-4): 287-291.

ALLUÉ ANDRADE, J.L. -1990- Atlas fitoclimático de España. Taxonomías. M.A.P.A. - I.N.I.A.

CABEZUDO,B., T. NAVARRO, A.V. PÉREZ LATORRE, J.M. NIETO CALDERA y G. ORSHAN. 1992. Estudios fenomorfológicos en la vegetación del sur de España. I. Cistus. Acta Bot. Malacitana, 17:2299-237.

CAIN, S.A. -1950- Life forms and phytoclimates. Bot. Rev., 16: 1-32.

CAPEL MOLINA, J.J. -1990- Climatología de Almería. Cuadernos Monográficos, 7: 1-160. Instituto de Estudios Almerienses. Almería.

MONTENEGRO, G., A.J. HOFFMANN, M.E. ALJARO \& A.E. HOFFMANN -1979-Satureja giliesii, apoiikilohydric shhrub from the Chilean mediterranean vegetation. Can. Journ. Bot., 57: 1206-1213.

ORSHAN, G \& G. ZAND -1962- Seasonal body reduction of certain desert halfshrubs. Bull.Res. Counc. Israel. 11D. Vol. 11.PP. 35-42

ORSHAN, G. -1982- Monocharacter growth formtypes as atool in an analytic-synthetic study of growth forms in mediterranean type ecosistems. A proposal for an inter-regional program. Ecol. mediterranea, 8: 159-171.
ORSHAN,G. -1964- Seasonal dimorphism of desert and mediterranean chamaephytes and their significance as a factor in their water economy. In: Rutter AJ.,Whitehead FH (eds.). Water in relation to plants. Blackwell, Oxford, pp 206-222.

ORSHAN, G.(ed.). -1989- Plant phenomorphological studies in Mediterranean type ecosystems. Kluwe Academic Publishers. Dodrecht.

PEINADO, M., F. ALCARAZ y J.M. MARTINEZ PARRAS -1992-Vegetation of Sautheastern Spain. Flora et Vegetatio Mundi. Band. X. 1992. J. Cramer. Berlin Stuttgart.

RAUNKIAER, C. -1934- Life forms of plants and statistical plant geography. Oxford, clarendon press.

Aceptado para su publicación en Julio de 1993

Dirección de los autores. Departamento de Biología Vegetal Facultad de Ciencias. Universidad de Málaga. 29080, Málaga. 